\title{
Energy and momentum flux in a high-numerical-aperture beam using the extended Nijboer-Zernike diffraction formalism
}

Joseph J. M. Braat

j.j.m.braat@tudelft.nl

Sven van Haver

s.vanhaver@tudelft.nl

Augustus J. E. M. Janssen

a.j.e.m.janssen@philips.com

Peter Dirksen

peter.dirksen@philips.com

\author{
Optics Research Group, Faculty of Applied Sciences, Technical University Delft, Lorentzweg 1, NL- \\ 2628 CJ Delft, The Netherlands \\ Optics Research Group, Faculty of Applied Sciences, Technical University Delft, Lorentzweg 1, NL- \\ 2628 CJ Delft, The Netherlands \\ Philips Research Europe, HTC 36 / 4 , NL-5656 AE Eindhoven, The Netherlands
}

Philips Research Europe, HTC 36 / 4 , NL-5656 AE Eindhoven, The Netherlands

We describe the energy and momentum flux in the case of an aberrated optical imaging system with a high numerical aperture (NA). The approach is based on the extended Nijboer-Zernike diffraction theory, that, in its high-NA version, yields an accurate analytic representation of the electromagnetic field vectors in the focal region of imaging systems that suffer from aberrations and/or transmission defects[1]. In an earlier publication, we have derived the electromagnetic energy density from the field vectors. In this paper, we expand our analysis to the energy flow (Poynting vector) and to the quantities related to the linear and angular momentum of the radiation. Several examples of the energy and momentum flow are presented. In particular, we show how the linear and angular momentum distribution in the focal region depend on the initial polarisation state and on the parameters describing the wavefront shape of the converging beam. For the angular momentum flow, we show how the separation between spin and orbital momentum is modified when going from the paraxial case to a high-NA focused beam. [DOI: 10.2971/jeos.2007.07032]

Keywords: Point-spread function, Poynting vector, linear and angular momentum, aberrations

\section{INTRODUCTION, EM-FIELD COMPONENTS AND CONSERVATION LAWS}

The electromagnetic field in focus has been studied for imaging systems with a high numerical aperture[2,3]. The source was pointlike, located at a large distance and supposed to emit a virtually planar wave towards the entrance pupil of the system; the state of polarisation was linear in these papers. Further research has focused on the effects of aberrations and on more general states of polarisation [4]-[11]. The expressions for the field in focus comprise integrals that have to be evaluated numerically. In the above mentioned previous publication [1], well-converging analytic series expansions from the extended Nijboer-Zernike theory $[12,13]$ were used to obtain the energy density in the focal region of an aberrated optical system. The energy density is the relevant quantity when media like photoresist in lithography or Si-based CCD-detectors are used for recording the image information. In this paper, we will concentrate on other electromagnetic quantities that may carry information in an imaging system. To this goal we will study the energy flow (Poynting vector) and the quantities that relate to the linear and angular momentum of a (focused) light beam. The basic quantities that one needs to produce the energy and momentum distribution in the focal region of a high-NA imaging system are the electric and magnetic field vectors. Once the vector components of the electric and magnetic field vectors have been obtained, the energy flux, momentum density and corresponding flux components can be derived. In this section we will first briefly recall the expressions for the electric and magnetic field vectors in the focal region, for a general state of polarisation and for arbitrary aberration. In Section 2, the analytic expressions for the energy flow components, the Poynting vector, are derived. We use the $G_{k l}$-functions [14], $|k|,|l| \leq 2$, that, in the case of a system with arbitrary aberration, replace the $I_{0^{-}}, I_{1^{-}}$and $I_{2^{-}}$ integrals that were developed in the paper by Richards and Wolf [3] for the aberration-free case. Section 3 focuses on the linear and angular momentum densities and the corresponding flow components. Here too, we will use the $G_{k l}$-functions to present our analytic results. Finally, in Section 4, we present some numerical examples that illustrate flow patterns in highnumerical-aperture beams for some typical states of polarisation and types of aberration. Section 5 presents the conclusions of our work.

\subsection{The electric and magnetic field components}

Expressions have been given for the Cartesian electric field components in the focal region of a high-NA system in the presence of a linear state of polarisation in the entrance pupil, 
either along the $x$ - or $y$-direction, $\mathbf{E}^{x}$ and $\mathbf{E}^{y}$, respectively [1]. The coordinate systems and imaging geometry that are used in this paper are sketched in Figure 1. A general elliptical

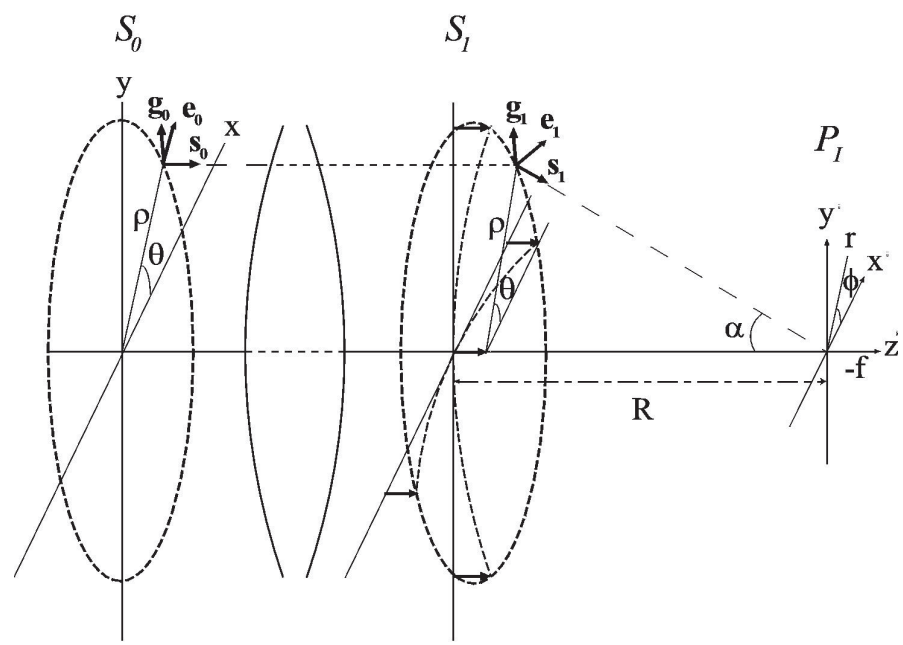

FIC. 1 The propagation of the incident wave from the entrance pupil $S_{0}$ through the optical system towards the exit pupil $S_{1}$ and the focal region at the image plane $P_{I}$. The incident wave has a planar wave front. The unit propagation vector has been denoted by $\mathbf{s}_{0}$, the meridional and tangential field components are directed along, respectively, the unit vectors $\mathbf{e}_{0}$ and $\mathbf{g}_{0}$. After propagation through the optical system, the field components in the exit pupil are projected onto the unit vectors $\mathbf{e}_{\mathbf{1}}$ and $\mathbf{g}_{\mathbf{1}}$, that form an orthogonal basis with the local propagation vector $\mathbf{s}_{\mathbf{1}}$. The position on the exit pupil sphere is defined by means of the cylindrical coordinates $(\rho, \theta)$ with the radial coordinate $\rho$ normalised to unity; the position in the image plane region is defined by the (normalised) cylindrical coordinate system $(r, \phi, f)$. The maximum aperture (NA) of the imaging pencil is represented by $s_{0}=\sin \alpha_{\max }$.

state of polarisation in the entrance pupil can be described by multiplying the $x$-and $y$-components in the entrance pupil with complex numbers $a$ and $b$, respectively, and summing the vector components in the high-NA focal region with these weighting factors. A lengthy but straightforward calculation yields the following result result for the electric vector

$$
\begin{gathered}
\mathbf{E}(r, \phi, f)=-i \gamma s_{0}^{2} \exp \left[\frac{-i f}{u_{0}}\right] \sum_{n, m} i^{m} \beta_{n}^{m} \exp [i m \phi] \times \\
\left(\begin{array}{c}
a V_{n, 0}^{m}+\frac{s_{0}^{2}}{2}\left\{(a-i b) V_{n, 2}^{m} \exp [2 i \phi]\right. \\
\left.+(a+i b) V_{n,-2}^{m} \exp [-2 i \phi]\right\} \\
b V_{n, 0}^{m}-i \frac{s_{0}^{2}}{2}\left\{(a-i b) V_{n, 2}^{m} \exp [2 i \phi]\right. \\
\left.-(a+i b) V_{n,-2}^{m} \exp [-2 i \phi]\right\} \\
-i s_{0}\left\{(a-i b) V_{n, 1}^{m} \exp [i \phi]-(a+i b) V_{n,-1}^{m} \exp [-i \phi]\right\}
\end{array}\right)
\end{gathered}
$$

Here, we have used the normalised cylindrical coordinates $r, \phi, f$ in the image space. They have been derived from the real-space Cartesian coordinates $\left(x^{\prime}, y^{\prime}, z^{\prime}\right)$ according to

$$
\begin{aligned}
r & =\frac{s_{0}}{\lambda} \sqrt{\left(x^{\prime}\right)^{2}+\left(y^{\prime}\right)^{2},} \\
f & =-2 \pi z u_{0} / \lambda, \\
u_{0} & =1-\sqrt{1-s_{0}^{2}}=1-\cos \alpha,
\end{aligned}
$$

with $s_{0}=\sin \alpha$ the geometrical aperture of the focusing beam. The defocus parameter $f$ is orientated in the opposite sense as compared to the real-space coordinate $z^{\prime}$ and equals zero in the geometrical focus of the beam. The reason for the sign difference between $f$ and $z^{\prime}$ is the difference in phase advance between an obliquely propagating plane wave component and an axial plane wave if the phase reference point is shifted in the positive $z^{\prime}$-direction; this phase difference becomes negative in the case of a positive $z^{\prime}$-increment. The factor $\gamma$, a proportionality constant, is given by $\gamma=\frac{\pi R}{\lambda}$. The $\beta_{n}^{m}$-coefficients are related to the complex transmission function (pupil function) of the imaging system according to

$$
\begin{aligned}
P^{x}(\rho, \theta) & =A^{x}(\rho, \theta) \exp [i 2 \pi W(\rho, \theta)] \\
& =a \sum_{n, m} \beta_{n}^{m} R_{n}^{|m|}(\rho) \exp \{i m \theta\}, \\
P^{y}(\rho, \theta) & =A^{y}(\rho, \theta) \exp [i 2 \pi W(\rho, \theta)] \\
& =b \sum_{n, m} \beta_{n}^{m} R_{n}^{|m|}(\rho) \exp \{i m \theta\},
\end{aligned}
$$

where $a$ and $b$ are normalised complex factors with $|a|^{2}+$ $|b|^{2}=1$ that determine the incident state of polarisation and $R_{n}^{|m|}(\rho)$ is a radial Zernike polynomial of radial degree $n$ and azimuthal order $m$.

The functions $V_{n, j}^{m}$ that depend on the normalised radial coordinate $r$ and the defocus parameter $f$ are given by $(j=$ $-2,-1,0,1,2)$

$$
\begin{aligned}
& V_{n, j}^{m}(r, f)=\int_{0}^{1} \rho^{|j|} \frac{\left(1+\sqrt{1-s_{0}^{2} \rho^{2}}\right)^{-|j|+1}}{\left(1-s_{0}^{2} \rho^{2}\right)^{1 / 4}} \\
& \times \exp \left[\frac{i f}{u_{0}}\left(1-\sqrt{1-s_{0}^{2} \rho^{2}}\right)\right] R_{n}^{|m|}(\rho) J_{m+j}(2 \pi r \rho) \rho d \rho .
\end{aligned}
$$

Expressions with series expansions are available to obtain quick and accurate values of the integral in Eq.(5), see [1, 15].

The magnetic induction components $\mathbf{B}$ are obtained from the electric components according to

$$
\mathbf{B}=n_{r} \frac{\mathbf{s} \times \mathbf{E}}{c},
$$

where $\mathbf{s}$ is the unit vector from a point in the exit pupil to the focal plane and $c / n_{r}$ is the speed of propagation of the light. The expressions for $\mathbf{E}$ and $\mathbf{B}$ are good approximations for weakly aberrated systems where the direction of the Poynting vector deviates only slightly from that in a perfect imaging system. Using Eq.(1) the components of the magnetic induction vector are written as

$$
\begin{gathered}
\mathbf{B}(r, \phi, f)=-\frac{i n_{r} \gamma s_{0}^{2}}{c} \exp \left[\frac{-i f}{u_{0}}\right] \sum_{n, m} i^{m} \beta_{n}^{m} \exp [i m \phi] \times \\
\left(\begin{array}{c}
-b V_{n, 0}^{m}-i \frac{s_{0}^{2}}{2}\left\{(a-i b) V_{n, 2}^{m} \exp [2 i \phi]\right. \\
\left.-(a+i b) V_{n,-2}^{m} \exp [-2 i \phi]\right\} \\
a V_{n, 0}^{m}-\frac{s_{0}^{2}}{2}\left\{(a-i b) V_{n, 2}^{m} \exp [2 i \phi]\right. \\
\left.+(a+i b) V_{n,-2}^{m} \exp [-2 i \phi]\right\} \\
-s_{0}\left\{(a-i b) V_{n, 1}^{m} \exp [i \phi]+(a+i b) V_{n,-1}^{m} \exp [-i \phi]\right\}
\end{array}\right)
\end{gathered}
$$




\subsection{The energy and momentum density and their respective flow components}

In this subsection, we briefly recall the definition of the energy and momentum density and the energy and momentum flow components in an isotropic and homogeneous medium. The electromagnetic energy density $w_{e m}$ is the sum of the electric and magnetic energy densities $w_{e}$ and $w_{m}$, respectively. The flow of electromagnetic energy is given by the components of the Poynting vector $\vec{S}$. In differential form, conservation of energy is described by

$$
\frac{\partial w_{e m}}{\partial t}+\nabla \cdot \vec{S}=0
$$

The linear momentum density of the electromagnetic field is a vector $\vec{m}$. The flow of momentum is given by the Maxwell stress tensor $\mathbf{T}$ whose entries $T_{i j}$ with $i, j=(x, y, z)$ have the dimension of pressure. The value of $T_{i j}$ yields the transport of the $i$-component of the linear momentum vector in the direction $j$. The diagonal elements of the stress tensor are called the normal pressure components, the off-diagonal elements the shear pressure components. The conservation of a linear momentum component $i$ is given by

$$
\frac{\partial m_{i}}{\partial t}+\frac{\partial T_{i x}}{\partial x^{\prime}}+\frac{\partial T_{i y}}{\partial y^{\prime}}+\frac{\partial T_{i z}}{\partial z^{\prime}}=0,
$$

where we have used real-space Cartesian coordinates $\left(x^{\prime}, y^{\prime}, z^{\prime}\right)$. In our normalised system of coordinates the conservation law reads

$$
\frac{\partial m_{i}}{\partial t}+\frac{s_{0}}{\lambda}\left[\frac{\partial T_{i x}}{\partial x}+\frac{\partial T_{i y}}{\partial y}\right]-\frac{2 \pi\left[1-\sqrt{1-s_{0}^{2}}\right]}{\lambda} \frac{\partial T_{i f}}{\partial f}=0,
$$

where the quantities have now been expressed using the normalised coordinates $(x, y, f)$.

In a comparable way, we define the angular momentum density $\vec{j}$ and the corresponding tensor $\mathbf{M}$ with the flow components $M_{i j}$. The conservation of angular momentum is then expressed as

$$
\frac{\partial j_{i}}{\partial t}+\frac{s_{0}}{\lambda}\left[\frac{\partial M_{i x}}{\partial x}+\frac{\partial M_{i y}}{\partial y}\right]-\frac{2 \pi\left[1-\sqrt{1-s_{0}^{2}}\right]}{\lambda} \frac{\partial M_{i f}}{\partial f}=0 .
$$

In what follows, we will successively produce expressions for the Poynting vector and the flow density components of linear and angular momentum. With the aid of the general expressions for the electric and magnetic field vectors in the focal region of an aberrated optical system, it is possible to develop semi-analytic expressions for the energy and momentum density and their flow components.

\section{ANALYTIC REPRESENTATION OF THE POYNTING VECTOR COMPONENTS}

To examine the energy flow through the focal region, the time averaged value of the Cartesian components of the Poynting vector $\mathbf{S}$ has to be found. The expression for the time averaged Poynting vector is given by

$$
\langle\mathbf{S}\rangle=\frac{\epsilon_{0} c^{2}}{2} \Re\left\{\mathbf{E} \times \mathbf{B}^{*}\right\} .
$$

We introduce the vector $\mathbf{p}=\left[c /\left(n_{r} \gamma^{2} s_{0}^{4}\right]\left(\mathbf{E} \times \mathbf{B}^{*}\right)\right.$ and, using Eqs.(1) and (7) for the electromagnetic field vectors, we obtain, after some lengthy manipulation, the following expressions for the components $p_{x}, p_{y}$ and $p_{z}$,

$$
\begin{aligned}
& p_{x}=s_{0}\left\{-2 i a^{*} b\left[\Im\left(G_{0,1}\right)+\Im\left(G_{0,-1}\right)\right]\right. \\
& \left.+i\left\{|a|^{2}\left[G_{1,0}-G_{-1,0}\right]-|b|^{2}\left[G_{0,1}-G_{0,-1}\right]\right\}\right\} \\
& -s_{0}^{3}\left[\left\{-2 \Im\left(a b^{*}\right)+|a|^{2}+|b|^{2}\right\} \Im\left(G_{2,1}\right)\right. \\
& -\left\{2 \Im\left(a b^{*}\right)+|a|^{2}+|b|^{2}\right\} \Im\left(G_{-2,-1}\right) \\
& +2 i \Re\left(a b^{*}\right) \Im\left(G_{1,-2}-G_{2,-1}\right) \\
& \left.+i\left(|a|^{2}-|b|^{2}\right) \Re\left(G_{1,-2}-G_{2,-1}\right)\right] \text {, } \\
& p_{y}=s_{0}\left\{2 i a b^{*} \Re\left[G_{0,1}-G_{0,-1}\right]\right. \\
& \left.+|a|^{2}\left[G_{0,1}+G_{0,-1}\right]+|b|^{2}\left[G_{1,0}+G_{-1,0}\right]\right\} \\
& +s_{0}^{3}\left[\left\{-2 \Im\left(a b^{*}\right)+|a|^{2}+|b|^{2}\right\} \Re\left(G_{2,1}\right)\right. \\
& +\left\{2 \Im\left(a b^{*}\right)+|a|^{2}+|b|^{2}\right\} \Re\left(G_{-2,-1}\right) \\
& +2 i \Re\left(a b^{*}\right) \Re\left(G_{1,-2}-G_{2,-1}\right) \\
& \left.-i\left(|a|^{2}-|b|^{2}\right) \Im\left(G_{1,-2}-G_{2,-1}\right)\right],
\end{aligned}
$$

$$
\begin{aligned}
p_{z}= & \left(|a|^{2}+|b|^{2}\right) G_{0,0} \\
& -s_{0}^{2}\left[i\left(|a|^{2}-|b|^{2}\right) \Im\left(G_{0,2}\right)+2 i \Re\left(a b^{*}\right) \Re\left(G_{0,2}\right)\right. \\
& \left.+i\left(|a|^{2}-|b|^{2}\right) \Im\left(G_{0,-2}\right)-2 i \Re\left(a b^{*}\right) \Re\left(G_{0,-2}\right)\right] \\
& -\frac{s_{0}^{4}}{2}\left[\left\{|a|^{2}+|b|^{2}-2 \Im\left(a b^{*}\right)\right\} G_{2,2}\right. \\
& \left.+\left\{|a|^{2}+|b|^{2}+2 \Im\left(a b^{*}\right)\right\} G_{-2,-2}\right] .
\end{aligned}
$$

Here we have used the functions $G_{k, l}$ that, in complete notation, are given by

$$
\begin{aligned}
G_{k l}(\beta)= & \sum_{n, m} i^{m} \exp [i m \phi] \beta_{n}^{m} V_{n, k}^{m}(r, f) \exp [i k \phi] \\
& \times \sum_{n^{\prime}, m^{\prime}} i^{-m^{\prime}} \exp \left[-i m^{\prime} \phi\right] \beta_{n^{\prime}}^{m^{\prime}} * V_{n^{\prime}, l}^{m^{\prime}} *(r, f) \exp [-i l \phi] \\
= & \sum_{n, m, n^{\prime}, m^{\prime}} \exp \left[i\left(m-m^{\prime}\right) \pi / 2\right] \exp \left[i\left(m-m^{\prime}+k-l\right) \phi\right] \\
& \times \beta_{n}^{m} \beta_{n^{\prime}}^{m^{\prime}} * V_{n, k}^{m}(r, f) V_{n^{\prime}, l}^{m^{\prime}} *(r, f) .
\end{aligned}
$$

The total number of $G_{k, l}$-functions is twenty-five and their general properties, $G_{k, l}=G_{l, k}^{*}, G_{k, k}$ is real, have been used 
in the derivations above. Fifteen independent $G$-functions occur in the general case with arbitrary pupil function. In the aberration-free case, the extra relationship $G_{-k,-k}=G_{k, k}$ applies, reducing the number of independent $G$-functions to thirteen.

To find the time-averaged energy flow and to eliminate the reactive part of the Poynting vector, the real parts of the above expressions for $p_{x}, p_{y}$ and $p_{z}$ should be inserted into the general expression of Eq.(12), leading to

$$
\begin{aligned}
& S_{x}= \frac{\epsilon_{0} c n_{r} \gamma^{2} s_{0}^{5}}{2}\left\{-2 \Im\left(a b^{*}\right) \Im\left(G_{0,1}+G_{0,-1}\right)\right. \\
&+\left(|a|^{2}+|b|^{2}\right) \Im\left(G_{0,1}-G_{0,-1}\right) \\
&+s_{0}^{2}\left[2 \Im\left(a b^{*}\right) \Im\left(G_{2,1}+G_{-2,-1}\right)\right. \\
&\left.\left.-\left(|a|^{2}+|b|^{2}\right) \Im\left(G_{2,1}-G_{-2,-1}\right)\right]\right\} \\
& S_{y}= \frac{\epsilon_{0} c n_{r} \gamma^{2} s_{0}^{5}\left\{-2 \Im\left(a b^{*}\right) \Re\left(G_{0,1}-G_{0,-1}\right)\right.}{2} \\
&+\left(|a|^{2}+\mid b^{2}\right) \Re\left(G_{0,1}+G_{0,-1}\right) \\
&+s_{0}^{2}\left[-2 \Im\left(a b^{*}\right) \Re\left(G_{2,1}-G_{-2,-1}\right)\right. \\
&\left.\left.+\left(|a|^{2}+|b|^{2}\right) \Re\left(G_{2,1}+G_{-2,-1}\right)\right]\right\} \\
& S_{z}=\frac{\epsilon_{0} c n_{r} \gamma^{2} s_{0}^{4}}{2}\left\{\left(|a|^{2}+|b|^{2}\right)\left[G_{0,0}-\frac{s_{0}^{4}}{2}\left(G_{2,2}-G_{-2,-2}\right)\right]\right. \\
&\left.+s_{0}^{4} \Im_{\left.\left(a b^{*}\right)\left(G_{2,2}-G_{-2,-2}\right)\right\} .}\right\}
\end{aligned}
$$

\subsection{The aberration-free system as a special case}

It is interesting to reduce the general expressions for the Poynting vector components to its simpler form in the case of an aberration-free imaging system. In that case, all $\beta_{n}^{m}$ are zero except $\beta_{0}^{0}$ which equals 1 . The $G$-functions reduce to

$$
G_{k l}(\beta)=\exp \{i(k-l) \phi\} V_{0, k}^{0}(r, f) V_{0, l}^{0 *}(r, f),
$$

After some manipulation, the Cartesian Poynting vector components are found to be

$$
\begin{aligned}
S_{x}= & \epsilon_{0} c n_{r} \gamma^{2} s_{0}^{5}\left\{\left[2 \Im\left(a b^{*}\right) \Re\left\{V_{0,0}^{0}(r, f) V_{0,1}^{0 *}(r, f)\right\} \sin \phi\right.\right. \\
& \left.+\left(|a|^{2}+|b|^{2}\right) \Im\left\{V_{0,0}^{0}(r, f) V_{0,1}^{0 *}(r, f)\right\} \cos \phi\right] \\
& +s_{0}^{2}\left[2 \Im\left(a b^{*}\right) \Re\left\{V_{0,2}^{0}(r, f) V_{0,1}^{0 *}(r, f)\right\} \sin \phi\right. \\
& \left.\left.-\left(|a|^{2}+|b|^{2}\right) \Im\left\{V_{0,2}^{0}(r, f) V_{0,1}^{0 *}(r, f)\right\} \cos \phi\right]\right\},
\end{aligned}
$$

$$
\begin{aligned}
S_{y}= & \epsilon_{0} c n_{r} \gamma^{2} s_{0}^{5}\left\{\left[-2 \Im\left(a b^{*}\right) \Re\left\{V_{0,0}^{0}(r, f) V_{0,1}^{0 *}(r, f)\right\} \cos \phi\right.\right. \\
& \left.+\left(|a|^{2}+|b|^{2}\right) \Im\left\{V_{0,0}^{0}(r, f) V_{0,1}^{0 *}(r, f)\right\} \sin \phi\right] \\
& -s_{0}^{2}\left[2 \Im\left(a b^{*}\right) \Re\left\{V_{0,2}^{0}(r, f) V_{0,1}^{0 *}(r, f)\right\} \cos \phi\right. \\
& \left.\left.+\left(|a|^{2}+|b|^{2}\right) \Im\left\{V_{0,2}^{0}(r, f) V_{0,1}^{0 *}(r, f)\right\} \sin \phi\right]\right\},
\end{aligned}
$$

$$
S_{z}=\frac{\epsilon_{0} c n_{r} \gamma^{2} s_{0}^{4}}{2}\left(|a|^{2}+|b|^{2}\right)\left\{\left|V_{0,0}^{0}(r, f)\right|^{2}-s_{0}^{4}\left|V_{0,2}^{0}(r, f)\right|^{2}\right\}
$$

Because of the basic circular symmetry of many diffraction problems, it is appropriate to use the cylindrical components of the Poynting vector with $S_{r}=S_{x} \cos \phi+S_{y} \sin \phi$ and $S_{\phi}=$ $-S_{x} \sin \phi+S_{y} \cos \phi$; this yields

$$
\begin{aligned}
S_{r}= & \epsilon_{0} c n_{r} \gamma^{2} s_{0}^{5}\left(|a|^{2}+|b|^{2}\right) \\
& \times \Im\left[V_{0,0}^{0}(r, f) V_{0,1}^{0 *}(r, f)-s_{0}^{2} V_{0,2}^{0}(r, f) V_{0,1}^{0 *}(r, f)\right],
\end{aligned}
$$

$$
\begin{aligned}
S_{\phi}= & -2 \epsilon_{0} c n_{r} \gamma^{2} s_{0}^{5} \\
& \Im\left(a b^{*}\right) \Re\left[V_{0,0}^{0}(r, f) V_{0,1}^{0 *}(r, f)+s_{0}^{2} V_{0,2}^{0}(r, f) V_{0,1}^{0 *}(r, f)\right],
\end{aligned}
$$

$S_{z}=\frac{\epsilon_{0} c n_{r} \gamma^{2} s_{0}^{4}}{2}\left(|a|^{2}+|b|^{2}\right)\left\{\left|V_{0,0}^{0}(r, f)\right|^{2}-s_{0}^{4}\left|V_{0,2}^{0}(r, f)\right|^{2}\right\}$.

Some conclusions can be drawn from the above expressions. Using the property $V_{0, k}^{0}(r,-f) V_{0, l}^{0 *}(r,-f)=$ $V_{0, k}^{0 *}(r, f) V_{0, l}^{0}(r, f)$, we can state that

- On-axis $(r=0)$, the power flow is also directed along the axis of the beam.

- The radial component changes sign through focus.

- The radial and the $z$-component do not depend on the state of polarisation of the incident beam.

- The azimuthal component is only present if the incident radiation is not linearly polarised $(\arg (a) \neq \arg (b))$. This corresponds to the presence of angular momentum in the focused beam.

- The azimuthal component is maximum in the case of circularly polarised light.

- The azimuthal component maintains its sign on both sides of the optimum focus.

- For large $s_{0}$, all components can locally change sign with respect to their average values if the $(r, f)$-dependent function becomes zero. This can give rise to regions where the energy flow is in the negative $z$-direction and also to the appearance of vortices in the energy flow pattern. 
- In the low-aperture case, the z-component is the only nonzero one.

As it was mentioned, for instance, for the case of circular polarisation, an azimuthal flow component is present in the focal plane. On the optical axis, the azimuthal component is zero. In the direct vicinity of the axis, the energy flow shows a lefthanded component in the case of RC-polarised light suggesting that the focused beam of light possesses a certain amount of angular momentum. With the positive $z$-direction as the viewing direction, we observe that the rotation sense of the electric field vector is left-handed for RC-polarised light and this rotation sense is in accordance with the sign of the angular momentum of the electric field distribution in the focal region. In the following, we will further analyse the momentum transport in a focused aberrated beam by deriving expressions for the transport of linear and angular momentum; this is the subject of the next section.

\section{LINEAR AND ANGULAR MOMENTUM FLUX IN A FOCUSED LIGHT BEAM}

The linear and angular momentum density and their flux counterparts in a beam of light are related to the impulse and spin of the photons (linear and spin momentum) and to the geometrical structure of the beam (orbital angular momentum). In this section we present these quantities for a highnumerical-aperture beam and we discuss the representation and interpretation of the angular momentum distribution in such a beam.

\subsection{Linear momentum flow}

The expressions for the linear momentum density and its flux components are given by $\epsilon_{0} \mathbf{E} \times \mathbf{B}^{*}$ and $\mathbf{T}$, respectively, where we now limit ourselves to the vacuum case with $n_{r}=1$. The time-averaged elements $T_{i j}$ of the Maxwell stress tensor are given by

$$
\left(\begin{array}{cc}
\frac{1}{2}\left(\epsilon_{0}|\mathbf{E}|^{2}+\frac{1}{\mu_{0}}|\mathbf{B}|^{2}\right) & -\epsilon_{0} E_{x} E_{y}^{*}-\frac{1}{\mu_{0}} B_{x} B_{y}^{*} \\
-\epsilon_{0}\left|E_{x}\right|^{2}-\frac{1}{\mu_{0}}\left|B_{x}\right|^{2} & \frac{1}{2}\left(\epsilon_{0}|\mathbf{E}|^{2}+\frac{1}{\mu_{0}}|\mathbf{B}|^{2}\right) \\
-\epsilon_{0} E_{y} E_{x}^{*}-\frac{1}{\mu_{0}} B_{y} B_{x}^{*} & -\epsilon_{0}\left|E_{y}\right|^{2}-\frac{1}{\mu_{0}}\left|B_{y}\right|^{2} \\
-\epsilon_{0} E_{z} E_{x}^{*}-\frac{1}{\mu_{0}} B_{z} B_{x}^{*} & -\epsilon_{0} E_{z} E_{y}^{*}-\frac{1}{\mu_{0}} B_{z} B_{y}^{*} \\
\\
-\epsilon_{0} E_{x} E_{z}^{*}-\frac{1}{\mu_{0}} B_{x} B_{z}^{*} \\
-\epsilon_{0} E_{y} E_{z}^{*}-\frac{1}{\mu_{0}} B_{y} B_{z}^{*} \\
\frac{1}{2}\left(\epsilon_{0}|\mathbf{E}|^{2}+\frac{1}{\mu_{0}}|\mathbf{B}|^{2}\right) \\
-\epsilon_{0}\left|E_{z}\right|^{2}-\frac{1}{\mu_{0}}\left|B_{z}\right|^{2}
\end{array}\right)
$$

The physical interpretation of the Maxwell stress tensor and its symmetry properties are discussed in various textbooks, see [16]. We now give the expressions for the transport of $x$, $y$ and $z$-linear momentum in the three orthogonal directions for a focused beam with arbitrary aperture by evaluating the stress tensor element $T_{i j}$. Taking the time-averaged real parts of the elements of expression (27) we obtain

$$
\begin{aligned}
& T_{x x}=\epsilon_{0} \gamma^{2} s_{0}^{6}\left\{\left(|a|^{2}+|b|^{2}\right)\left[G_{1,1}+G_{-1,-1}-\Re\left(G_{2,0}+G_{-2,0}\right)\right]\right. \\
& \left.-2 \Im\left(a b^{*}\right)\left[\left(G_{1,1}-G_{-1,-1}\right)-\Re\left(G_{2,0}-G_{-2,0}\right)\right]\right\} \text {, } \\
& T_{x y}=\epsilon_{0} \gamma^{2} s_{0}^{6}\left\{-\left(|a|^{2}+|b|^{2}\right) \Im\left(G_{2,0}-G_{-2,0}\right)\right. \\
& \left.+2 \Im\left(a b^{*}\right) \Im\left(G_{2,0}+G_{-2,0}\right)\right\} \text {, } \\
& T_{x z}=\epsilon_{0} \gamma^{2} s_{0}^{5}\left\{-\left(|a|^{2}+|b|^{2}\right)\right. \\
& \Im\left[G_{1,0}-G_{-1,0}+s_{0}^{2}\left(G_{1,2}-G_{-1,-2}\right)\right] \\
& \left.+2 \Im\left(a b^{*}\right) \Im\left[\left(G_{1,0}+G_{-1,0}\right)+s_{0}^{2}\left(G_{1,2}+G_{-1,-2}\right)\right]\right\}, \\
& T_{y x}=T_{x y}, \\
& T_{y y}=\epsilon_{0} \gamma^{2} s_{0}^{6}\left\{\left(|a|^{2}+|b|^{2}\right)\left[G_{1,1}+G_{-1,-1}+\Re\left(G_{2,0}+G_{-2,0}\right)\right]\right. \\
& \left.-2 \Im\left(a b^{*}\right)\left[\left(G_{1,1}-G_{-1,-1}\right)+\Re\left(G_{2,0}-G_{-2,0}\right)\right]\right\} \text {, } \\
& T_{y z}=\epsilon_{0} \gamma^{2} s_{0}^{5}\left\{\left(|a|^{2}+|b|^{2}\right) \Re\left[G_{1,0}+G_{-1,0}-s_{0}^{2}\left(G_{1,2}+G_{-1,-2}\right)\right]\right. \\
& \left.-2 \Im\left(a b^{*}\right) \Re\left[\left(G_{1,0}-G_{-1,0}\right)-s_{0}^{2}\left(G_{1,2}-G_{-1,-2}\right)\right]\right\}, \\
& T_{z x}=T_{x z} \\
& T_{z y}=T_{y z} \\
& T_{z z}=\epsilon_{0} \gamma^{2} s_{0}^{4}\left\{( | a | ^ { 2 } + | b | ^ { 2 } ) \left[G_{0,0}-s_{0}^{2}\left(G_{1,1}+G_{-1,-1}\right)\right.\right. \\
& \left.+\frac{s_{0}^{4}}{2}\left(G_{2,2}+G_{-2,-2}\right)\right]+2 \Im\left(a b^{*}\right) \\
& \left.\times\left[s_{0}^{2}\left(G_{1,1}-G_{-1,-1}\right)-\frac{s_{0}^{4}}{2}\left(G_{2,2}-G_{-2,-2}\right)\right]\right\} \text {. }
\end{aligned}
$$

For the aberration-free case, the generally dominating $T_{z j}$ flow components are given by

$$
\begin{aligned}
T_{z x}= & -2 \epsilon_{0} \gamma^{2} s_{0}^{5}\left\{\left(|a|^{2}+|b|^{2}\right) \Im\left[V_{0,1}^{0}\left(V_{0,0}^{0}+s_{0}^{2} V_{0,2}^{0}\right)^{*}\right] \cos \phi\right. \\
& \left.-2 \Im\left(a b^{*}\right) \Re\left[V_{0,1}^{0}\left(V_{0,0}^{0}+s_{0}^{2} V_{0,2}^{0}\right)^{*}\right] \sin \phi\right\} \\
T_{z y}= & -2 \epsilon_{0} \gamma^{2} s_{0}^{5}\left\{\left(|a|^{2}+|b|^{2}\right) \Im\left[V_{0,1}^{0}\left(V_{0,0}^{0}-s_{0}^{2} V_{0,2}^{0}\right)^{*}\right] \sin \phi\right. \\
& \left.+2 \Im\left(a b^{*}\right) \Re\left[V_{0,1}^{0}\left(V_{0,0}^{0}-s_{0}^{2} V_{0,2}^{0}\right)^{*}\right] \cos \phi\right\}, \\
T_{z z}= & \epsilon_{0} \gamma^{2} s_{0}^{4}\left(|a|^{2}+|b|^{2}\right)\left\{\left|V_{0,0}^{0}\right|^{2}-2 s_{0}^{2}\left|V_{0,1}^{0}\right|^{2}+s_{0}^{4}\left|V_{0,2}^{0}\right|^{2}\right\} .
\end{aligned}
$$

\subsection{Angular momentum flow}

Angular momentum in a focused beam stems from intrinsic or spin momentum of the photons in the beam or from a special geometrical structure of the beam. The latter contribution is, for instance, known from paraxial Gauss-Laguerre laser beam modes; the expression for the phase of the mode contains a factor $\exp (i l \phi)$ with $l$ the azimuthal index of the mode. With the $\exp \{i(k z-\omega t)\}$ convention for a wave propagating in the positive $z$-direction, the shape of the 'wavefront corresponds to a left-handed screw for a positive value of $l$. More complicated phase structures are possible and can be described by means of a superposition of Gauss-Laguerre beams of various orders. A more general phase structure, including the discontinuity, can equally well be described by means of Zernike 
polynomials although the discontinuity will in general ask for a large number of terms to assure reasonable convergence. The angular momentum density with vector components $j_{i}$ is directly obtained from the Poynting vector

$$
\mathbf{j}=\epsilon_{0} \mathbf{r} \times\left(\mathbf{E} \times \mathbf{B}^{*}\right) .
$$

The momentum flux density tensor elements $M_{i j}$ bear a direct relationship to the Maxwell stress tensor elements $T_{i j}$, according to

$$
\mathbf{M}=\mathbf{r} \times \mathbf{T},
$$

in which the elements of $\mathbf{M}$ are evaluated using $M_{i j}=$ $\sum_{m n} \epsilon_{i m n} r_{m} T_{n j}$ with $\epsilon_{i m n}$ the Levi-Civita symbol and where, as before, $T_{n j}$ are the time-averaged values of the Maxwell stresstensor elements.

As an example we calculate the component $M_{z z}(r, f, \phi)$, the axial angular momentum flux density through a surface perpendicular to the $z$-axis. The resulting expression is

$$
M_{z z}=\frac{\lambda r}{s_{0}}\left[T_{y z} \cos \phi-T_{x z} \sin \phi\right],
$$

where we have used the relation $r=r^{\prime} s_{0} / \lambda$ between the realspace coordinate $r^{\prime}$ and the dimensionless coordinate $r$ (note, $\lambda=2 \pi c / \omega)$. The substitution of the tensor elements yields, after some rearrangement,

$$
\begin{aligned}
M_{z z}= & \epsilon_{0} \gamma^{2} s_{0}^{4} \lambda r\left[( | a | ^ { 2 } + | b | ^ { 2 } ) \left\{\Re \left[G_{1,0}+G_{-1,0}\right.\right.\right. \\
& \left.-s_{0}^{2}\left(G_{1,2}+G_{-1,-2}\right)\right] \cos \phi \\
& \left.+\Im\left[\left(G_{1,0}-G_{-1,0}\right)+s_{0}^{2}\left(G_{1,2}-G_{-1,-2}\right)\right] \sin \phi\right\} \\
& -2 \Im\left(a b^{*}\right)\left\{\Re\left[G_{1,0}-G_{-1,0}-s_{0}^{2}\left(G_{1,2}-G_{-1,-2}\right)\right] \cos \phi\right. \\
& \left.\left.+\Im\left[\left(G_{1,0}+G_{-1,0}\right)+s_{0}^{2}\left(G_{1,2}+G_{-1,-2}\right)\right] \sin \phi\right\}\right] .
\end{aligned}
$$

In the nominal focal plane (all functions $V_{n, k}^{m}$ are real) and in the absence of aberrations $\left(\beta_{0}^{0}=1\right.$, all other $\left.\beta_{n}^{m}=0\right)$, this expression reduces to

$$
M_{z z}=-4 \epsilon_{0} \gamma^{2} s_{0}^{4} \lambda r \Im\left(a b^{*}\right)\left[V_{0,1}^{0}\left(V_{0,0}^{0}-s_{0}^{2} V_{0,2}^{0}\right)\right] .
$$

The contribution to $M_{z z}$ stems from the spin momentum of the beam because it is proportional to $\Im\left(a b^{*}\right)$.

Another way to impart angular momentum to a beam is the introduction of a helical phase structure, e.g. by using the lowest order Zernike polynomial $\rho \exp \{+i \theta\}$, yielding so-called orbital angular momentum. In this case we approximate the exit pupil function by taking $\beta_{1}^{1} \equiv 1$ and equating all other $\beta$ coefficients to zero and, again in the nominal focal plane, we obtain the expression

$$
\begin{aligned}
M_{z z}= & \epsilon_{0} \gamma^{2} s_{0}^{4} \lambda\left\{( | a | ^ { 2 } + | b | ^ { 2 } ) \left[\left(V_{1,1}^{1}+V_{1,-1}^{1}\right) V_{1,0}^{1}\right.\right. \\
& \left.-s_{0}^{2}\left(V_{1,1}^{1} V_{1,2}^{1}+V_{1,-1}^{1} V_{1,-2}^{1}\right)\right] \\
& -2 \Im\left(a b^{*}\right)\left[\left(V_{1,1}^{1}-V_{1,-1}^{1}\right) V_{1,0}^{1}\right. \\
& \left.\left.-s_{0}^{2}\left(V_{1,1}^{1} V_{1,2}^{1}-V_{1,-1}^{1} V_{1,-2}^{1}\right)\right]\right\} .
\end{aligned}
$$

Although we would expect the orbital angular momentum associated with beam structure to be proportional to the power of the beam $\left(\alpha\left(|a|^{2}+|b|^{2}\right)\right)$, we observe a second term that is nonzero for elliptically polarised incident light beams. This relatively small term can be considered as a cross-term between spin and orbital angular momentum.

\subsection{Alternative representation of the axial angular momentum flux}

The flux component $M_{z z}$ has also been written in a different way [17] to make a distinction between the angular momentum contribution from the spin momentum and from the orbital momentum. This alternative notation for the integral $\int M_{z z} d x d y$ of the flux component $M_{z z}$ over a plane perpendicular to the $z$-axis is given by

$$
\begin{aligned}
& \int M_{z z} d x d y=\int \frac{\epsilon_{0} c^{2}}{2 \omega} \Re\left\{-i\left[\left(E_{x} B_{x}^{*}+E_{y} B_{y}^{*}\right)\right.\right. \\
& \left.\left.+\frac{1}{2}\left(-B_{x}^{*} \frac{\partial}{\partial \phi} E_{y}+E_{y} \frac{\partial}{\partial \phi} B_{x}^{*}-E_{x} \frac{\partial}{\partial \phi} B_{y}^{*}+B_{y}^{*} \frac{\partial}{\partial \phi} E_{x}\right)\right]\right\} d x d y,
\end{aligned}
$$

where $\omega$ is again the angular frequency of the monochromatic radiation. In the paraxial approximation, the contribution on the first line of Eq.(46) can be attributed to the spin momentum of the light while terms on the second line are caused by, for instance, a helical wavefront shape of the beam. The same separation between spin and orbital momentum remains possible for a non-paraxial beam with a fixed phase dependence $\exp (i m \phi)$, as shown in [17].

\subsubsection{Spin-induced angular momentum for an aberration-free system}

The spin-induced angular momentum component that has to be integrated can be directly obtained from Eq.(46) using the expressions from Eqs.(1) and (7). After a somewhat lengthy derivation, the substitution of the electric and magnetic field expressions yields the result

$$
\begin{aligned}
M_{z z}^{s}= & \frac{\epsilon_{0} c \gamma^{2} s_{0}^{4}}{2 \omega}\left\{-\Im\left(a b^{*}\right)\left[2 G_{0,0}+s_{0}^{4}\left(G_{2,2}+G_{-2,-2}\right)\right]\right. \\
& \left.+\frac{s_{0}^{4}}{2}\left(|a|^{2}+|b|^{2}\right)\left(G_{2,2}-G_{-2,-2}\right)\right\} .
\end{aligned}
$$

The term on the second line of Eq.(47) is independent of the state of polarisation of the incident light beam. In that sense, it can not be directly associated to the photon spin. It should be noted that it equals zero for an aberration-free beam. The term with the factor $\left(G_{2,2}-G_{-2,-2}\right)$ becomes nonzero when helical phase terms are present in the wavefront, that break the symmetry (or anti-symmetry) between the aberration coefficients with positive and negative upper index $m$. We conclude that the splitting in spin and orbital momentum that was suggested in Ref.[17], see also Eq.(46), remains applicable in the integral sense but can not be locally applied to the high-NA case in the presence of a general beam structure.

In the case of RC-polarised light $\left(\Im\left(a b^{*}\right)=1\right)$ and for an aberration-free imaging system $\left(\beta_{0}^{0}=1\right.$, all other $\left.\beta \equiv 0\right)$, we 
obtain

$$
M_{z z}^{s}=-\frac{\epsilon_{0} c \gamma^{2} s_{0}^{4}}{\omega}\left\{\left|V_{0,0}^{0}\right|^{2}+s_{0}^{4}\left|V_{0,2}^{0}\right|^{2}\right\},
$$

a negative value by convention. However, the expression is not in accordance with Eq.(44). The basic difference is the nonzero value on the optical axis. For values of $s_{0}<<1$, the expressions (44) and (48) can be analytically integrated over the nominal focal plane $(f=0)$. The resulting expressions are identical and this confirms the identity of (44) and (48) in the integral sense for the paraxial situation.

\subsubsection{Orbital angular momentum}

Continuing to adopt the separation between spin and orbital angular momentum according to Eq.(46), we need to evaluate the azimuthal derivatives of the electric field and magnetic induction vectors. In view of this, we introduce $G_{k l}^{(1,2)}$-functions according to

$$
\begin{aligned}
G_{k l}^{(1)}= & i \exp \{i(k-l) \phi\} \sum_{n, m, n^{\prime}, m^{\prime}}(m+k) \exp \left[i\left(m-m^{\prime}\right) \pi / 2\right] \\
& \times \exp \left[i\left(m-m^{\prime}\right) \phi\right] \beta_{n}^{m} \beta_{n^{\prime}}^{m^{\prime}} * V_{n, k}^{m}(r, f) V_{n^{\prime}, l}^{m^{\prime}} *(r, f), \\
G_{k l}^{(2)}= & -i \exp \{i(k-l) \phi\} \sum_{n, m, n^{\prime}, m^{\prime}}\left(m^{\prime}+l\right) \exp \left[i\left(m-m^{\prime}\right) \pi / 2\right] \\
& \times \exp \left[i\left(m-m^{\prime}\right) \phi\right] \beta_{n}^{m} \beta_{n^{\prime}}^{m^{\prime}} * V_{n, k}^{m}(r, f) V_{n^{\prime}, l}^{m^{\prime}} *(r, f),
\end{aligned}
$$

with, for instance, $E_{x} \partial B_{y}^{*} / \partial \phi$ using $G_{k l}^{(1)}$ and $\partial E_{x} / \partial \phi B_{y}^{*}$ the second function $G_{k l}^{(2)}$. From the definitions of $G_{k l}^{(1,2)}$ one readily derives the property

$$
G_{k l}^{(2)}=G_{l k}^{(1) *}
$$

that will be used to retain only the $G_{k l}^{(1)}$-function in the final expression for $M_{z z}^{0}$.

Starting with the expression on the second line of Eq.(46), we obtain after a somewhat lengthy manipulation

$$
\begin{aligned}
M_{z z}^{o}= & \frac{\epsilon_{0} c \gamma^{2} s_{0}^{4}}{4 \omega}\left[2\left(|a|^{2}+|b|^{2}\right) \Im\left(G_{0,0}^{(1)}\right)\right. \\
& -s_{0}^{4}\left\{\left(|a|^{2}+|b|^{2}\right) \Im\left(G_{2,2}^{(1)}+G_{-2,-2}^{(1)}\right)\right. \\
& \left.\left.-2 \Im\left(a b^{*}\right) \Im\left(G_{2,2}^{(1)}-G_{-2,-2}^{(1)}\right)\right\}\right] \\
= & \frac{\epsilon_{0} c \gamma^{2} s_{0}^{4}}{4 \omega}\left[\left(|a|^{2}+|b|^{2}\right) \Im\left\{2 G_{0,0}^{(1)}-s_{0}^{4}\left(G_{2,2}^{(1)}+G_{-2,-2}^{(1)}\right)\right\}\right. \\
& \left.+2 s_{0}^{4} \Im\left(a b^{*}\right) \Im\left\{G_{2,2}^{(1)}-G_{-2,-2}^{(1)}\right\}\right] .
\end{aligned}
$$

Note that the expression for the orbital angular momentum contains a term, on the second line of Eq.(52), that is only nonzero if the incident light is elliptically polarised.

Two special cases need a further analysis.

\subsubsection{Aberration-free case}

We first consider the aberration-free case with $\beta_{0}^{0} \equiv 1$ and all other $\beta$-coefficients equal to zero. In this particular case, $G_{k k}^{(1)}=i k\left|V_{0, k}^{0}(r, f)\right|^{2}$ is purely imaginary and we obtain

$$
M_{z z}^{o}=\frac{2 \epsilon_{0} c \gamma^{2} s_{0}^{8}}{\omega} \Im\left(a b^{*}\right)\left|V_{0,2}^{0}\right|^{2} .
$$

We conclude from the above expression that at high numerical aperture a term is present in the orbital angular momentum function that is connected to the spin momentum. Although relatively small (proportional to $s_{0}^{4}$ ), this term should be qualified as a cross-term between spin and orbital momentum.

\subsubsection{Helical wavefront}

Again representing the orbital beam structure by means of the substitution $\beta_{1}^{1}=1$, all other $\beta_{n}^{m}=0$, we obtain $G_{k, k}^{(1)}=$ $i(k+1)\left|V_{1, k}^{1}\right|^{2}$ and the orbital angular momentum expression according to

$$
\begin{aligned}
& M_{z z}^{o}=\frac{\epsilon_{0} c \gamma^{2} s_{0}^{4}}{4 \omega}\left[( | a | ^ { 2 } + | b | ^ { 2 } ) \left\{2\left|V_{1,0}^{1}\right|^{2}\right.\right. \\
& \left.\left.-s_{0}^{4}\left(3\left|V_{1,2}^{1}\right|^{2}-\left|V_{1,-2}^{1}\right|^{2}\right)\right\}+2 s_{0}^{4} \Im\left(a b^{*}\right)\left(3\left|V_{1,2}^{1}\right|^{2}+\left|V_{1,-2}^{1}\right|^{2}\right)\right] .
\end{aligned}
$$

In the paraxial regime the expression reduces to

$$
M_{z z}^{o}=\frac{\epsilon_{0} c \gamma^{2} s_{0}^{4}}{2 \omega}\left(|a|^{2}+|b|^{2}\right)\left|V_{1,0}^{1}\right|^{2}
$$

The flux of the z-oriented angular momentum does not show an azimuthal dependence over the beam cross-section and is only dependent on the radial coordinate. The angular momentum strength is independent of the state of polarisation of the beam; it only depends on $\left(|a|^{2}+|b|^{2}\right)$, the total power present in the two orthogonal states of polarisation. We emphasise that all considerations on separation between spin and orbital momentum are only valid in the integral sense, viz. after an integration over $\phi$ and over the complete range of $r$ from 0 to $\infty$.

\subsubsection{Assignment of angular momentum to spin and orbital origin in a high-NA focused beam}

Based on the analysis of the angular momentum representation of Eq.(46), we propose a subdivision between spin and orbital momentum for high-NA beams that is an extension of the paraxial case. Knowing that spin momentum, see Eq.(47), originates from ellipticity in the incident beam $\left(\Im\left(a b^{*}\right) \neq 0\right)$, we basically attribute the angular momentum terms that depend on this factor to spin momentum. However, in Eq.(47), we also observe a term that is proportional to the beam power $\left(|a|^{2}+|b|^{2}\right)$ only and that has a relative weight of $s_{0}^{4}$. This nonparaxial term is zero in the aberration-free case but, for instance with a helical beam structure, it constitutes a crossterm between spin and orbital momentum. Likewise, inspecting Eq.(52) for the orbital angular momentum, we observe a main term that is proportional to the beam power and only nonzero in the case of a helical phase aberration. On the last line of Eq.(52), a term is present that is proportional to $\Im\left(a b^{*}\right)$ and is nonzero for a beam with orbital structure via the $G_{2,2}^{(1)}$ and $G_{-2,-2}^{(1)}$-functions. This term again represents a cross-term between spin and orbital momentum.

\section{NUMERICAL EXAMPLES}

In this section, we present several examples of energy density and energy flux in the focal region. We also analyse the 
flux of linear and, more specifically, angular momentum, both as a function of the incident state of polarisation, the numerical aperture and the aberrations of the focused beam. Some unexpected phenomena will be highlighted. In order to have a hierarchy in strength of the focal field components, we have taken a fixed entrance pupil diameter, typical for a microscope objective, with a diameter $2 a=4 \mathrm{~mm}$. The power incident on the entrance pupil surface is $1 \mathrm{~mW}$. The focal distance of the objective is related to the chosen numerical aperture of the focusing 'device' according to $s_{0}=a / f$. This allows us to give absolute values of the electric and magnetic fields, of the magnitude of the Poynting vector and of the momentum density and flow components in the focal region. Because of the widely varying lateral scale and axial scale in the focal region as a function of the $s_{0}$-value, we have normalised the lateral and axial coordinates according to the prescription just below Eq.(1). All numerical results have been obtained using both the through-focus calculation of the electric and magnetic field components and the composite $G_{k l}$-quantities based on the extended Nijboer-Zernike diffraction theory.

\subsection{Energy density and energy flux}

We first present the energy density distribution and the Poynting vector components in the aberration-free case, both in focus $(f=0)$, two focal depths in front of the nominal focal plane $(f=-\pi)$ and two focal depths beyond this plane $(f=\pi)$. In Figure 2, upper row, we have plotted by means of arrows the $(x, y)$-components of the Poynting vector; the energy density has been represented by means of colour shading. In the lower row, the $z$-component of the Poynting vector has been plotted. The state of polarisation of the incident light is linear along the $x$-direction. The electric energy density shows the well-known elongated shape [3] due to the presence of an appreciable $z$-component of the electric field. A plot of the total electromagnetic energy density in vacuum would show circular symmetry because of the complementary distribution of the magnetic energy density. The z-component of the Poynting vector presents a circularly symmetric pattern, as predicted in the absence of aberrations by Eq.(26). This circular symmetry in the aberration-free case stems from the product operation of electric and magnetic field vectors that cancels the lack of circular symmetry in the individual vector components of $\mathbf{E}$ and $\mathbf{B}$. In certain annular regions of the central figure $(f=0)$, the $z$-component of the Poynting vector is slightly negative. This indicates the presence of strongly curved streamlines and, in the extreme case, vortices in the energy flow pattern.

The presence of angular momentum in the focused beam becomes apparent once the incident state of polarisation is changed to circular. In Figure 3 the state of polarisation is left-circular $(a, b)=(1, i) / \sqrt{2}$. The transverse flow pattern of the Poynting vector is right-handed as seen in the righthanded $x y z$-coordinate system used in this paper. This is in accordance with the common sign convention in defining the screw sense of a general elliptical state of polarisation. The corresponding figure for right-circular light is identical apart from the opposite screw sense.

In Figure 4 we have plotted some energy flux lines in the
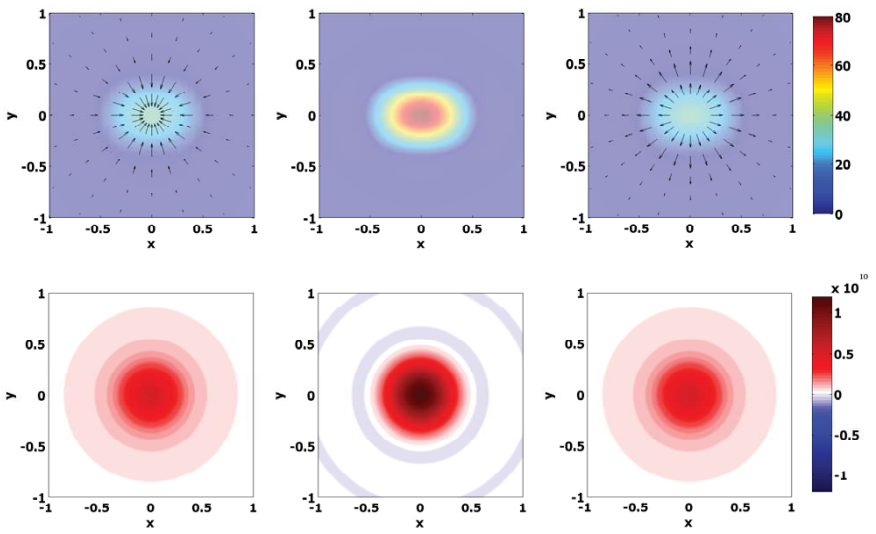

FIG. 2 Cross-sections of the energy density (upper row, colour-shading, units $\mathrm{Jm}^{-3}$ ) in three image plane settings (two focal depths in front of the nominal focal plane ( $f=\pi$ ), in focus $(f=0)$, and two focal depths beyond the nominal focal plane $(f=-\pi)$. In the same graphs, the $(x, y)$-components of the Poynting vector have been represented by arrows. The length of the arrows has been normalised to the largest transverse component in the picture. In the lower row, the $z$-component of the Poynting vector has been represented by means of colour shading (units $\mathrm{Wm}^{-2}$ ). The incident state of polarisation is linear ( $x$-direction), the numerical aperture of the focused beam is 0.95 , no aberration.
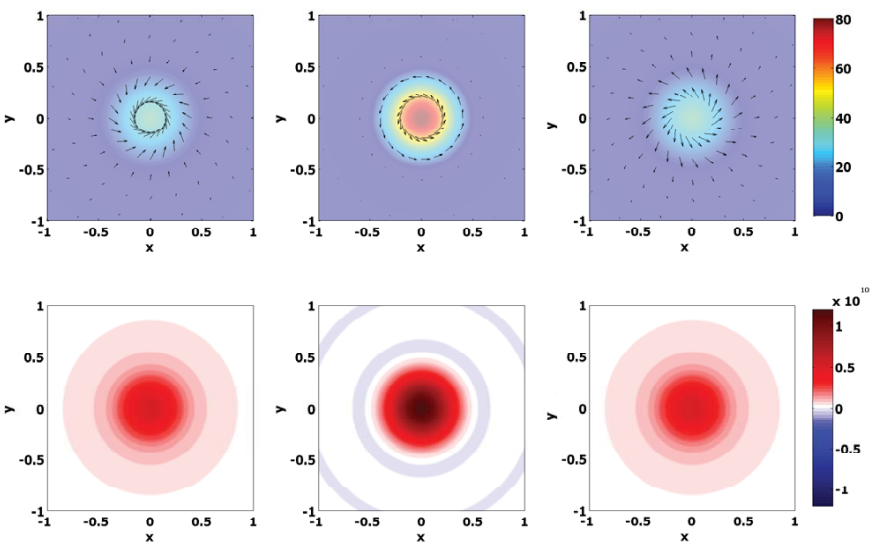

FIG. 3 Same legend as Figure 2, but the incident state of polarisation is now left-circular $(a, b)=(1, i) / \sqrt{2}$.

neighbourhood close to the zeros of the z-component of the Poynting vector. The flow pattern of the Poynting vector shows symmetry with respect to the optical axis (vertical in the figure) and there is a particular flow pattern around singular points, two of which have been represented in the figure, a vortex at $(r, f)=(0.55,0)$ and a saddle point structure at $(r, f)=(0.68,0)$. In Figure 5 the state of polarisation is linearly $x$-polarised, $(a, b)=(1,0)$, and the beam is affected by astigmatism with a set of Zernike aberration coefficients $\beta_{n}^{m}$ that correspond to an aberration $\Phi=\sum \alpha_{n}^{m} R_{n}^{m}(\rho) \exp (i m \theta)$ in the phase part of the pupil function $\exp \{i \Phi(\rho, \theta)\}$ with coefficients $\alpha_{2}^{2}=\alpha_{2}^{-2}=\pi / 4$ and all others equal to zero. The resulting $\beta$-coefficients were obtained using a third-order development of the exponential function according to $\exp (i \Phi)=$ $1+i \Phi-\Phi^{2} / 2-i \Phi^{3} / 6$. For certain low-order Zernike wavefront aberrations, it is also possible to obtain analytic expressions for the $\beta$-coefficients, see $[18,19]$. In focus, the electric energy density function has adopted the characteristic cross-like shape, while out-of-focus the characteristic focal lines become visible. The polarisation influence causes a disparity between 


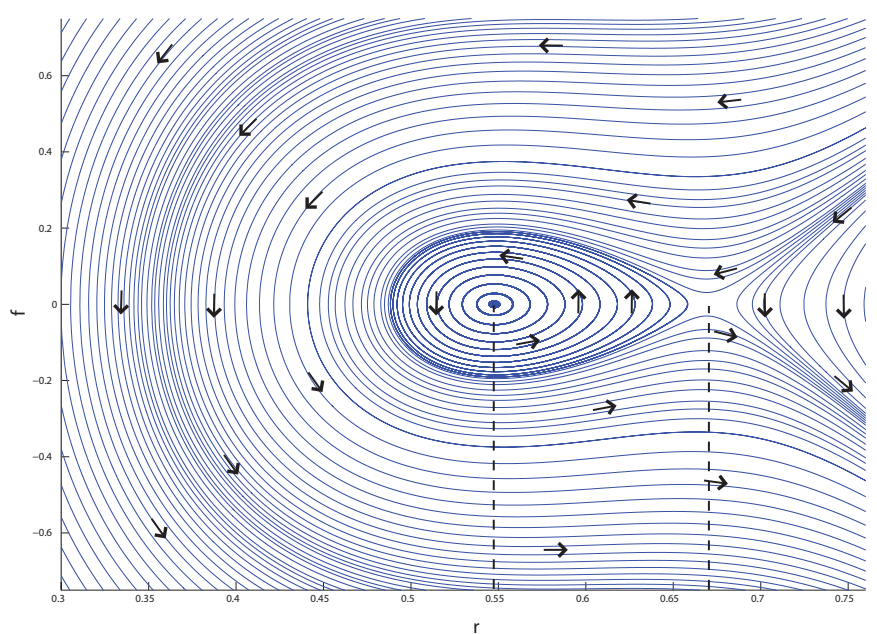

FIG. 4 Flow lines of the Poynting vector close to the first zeros in the nominal focal plane. The state of polarisation is arbitrary. The average propagation direction of the flow through focus is downward in the figure. Particular patterns in the energy flow pattern can be found, for instance, at lateral positions $r=0.55$ (vortex) and $r=0.68$ (saddle) in the nominal focal plane $(f=0.0)$.

the two focal lines. The transverse energy flow pattern shows the inward and outward direction in the out-of-focus planes; in focus, the flow pattern corresponding to a saddle-point is observed. The $z$-component of the Poynting vector shows the $4 \phi$-dependence in focus, out-of-focus the $2 \phi$-dependence is dominating.
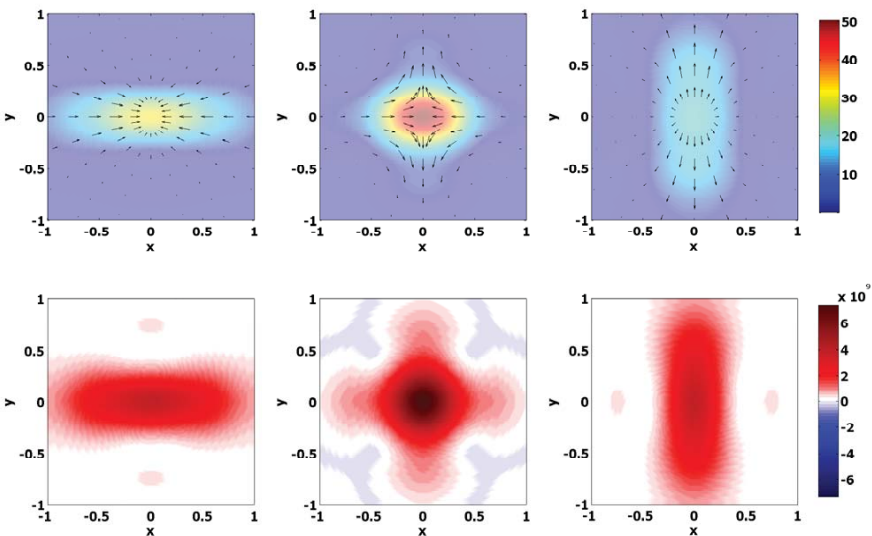

FIC. 5 Same legend as Figure 2, but now in the presence of a certain amount of astigmatic aberration.

\subsection{Linear and angular momentum flux}

In the following figures, we examine the particular behaviour of linear and angular momentum flux components in the focal region of a high-NA imaging system. A first analysis applies to a linearly polarised beam with the field vector in the entrance pupil oriented along the $x$-axis, $(a, b)=(1,0)$. In this first example, we keep the numerical aperture very low $\left(s_{0}=0.01\right)$ to establish the link with paraxial theory. The starting point of the analysis is given by the three components of the linear momentum density; in vacuum, they can be obtained from the Poynting vector by multiplication with a factor of $1 / c^{2}$. The stress tensor elements are represented in Figure 6 , rows one to three, where a dominating $T_{z z}$ flux com- ponent is visible. All other flux elements are small, especially $T_{x x}, T_{x y}, T_{y x}$ and $T_{y y}$, and their patterns remain invisible with the applied colour shading. Given the continuity relation of Eq.(10) for our static case, the partial $T$-derivatives have to be such that, for instance, $\frac{\partial T_{x x}}{\partial x}+\frac{\partial T_{x y}}{\partial y}=0$. A numerical check of the data represented in Figure 6 shows a tendency that corresponds to this general identity. Using Eq.(41) in general and Eq.(43) for the $M_{z z}$-component, we also produce a map of the angular momentum flux components in the nominal focal plane (rows four to six). The only appreciable components are $M_{x z}$ and $M_{y z}$ and, in combination, they give rise to a tangentially oriented flux component that varies with the radial coordinate $r$. Of course, the integration of the flux components of angular momentum over the entire focal plane yields zero. However, by local selection, we could extract a preferential radial momentum component, for instance, positive $x$ momentum flowing in the $z$-direction by selecting the halfplane $y>0$.
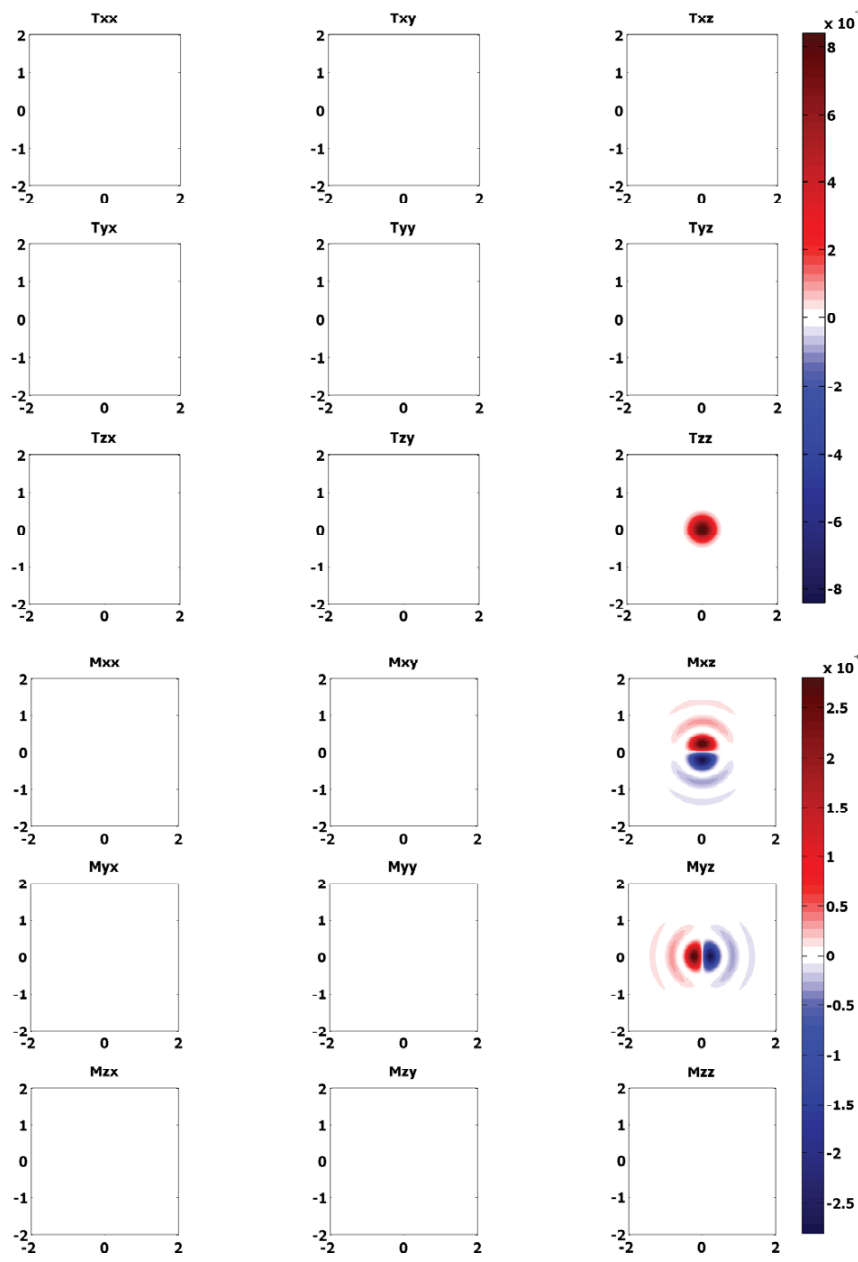

FIG. 6 The linear momentum flux components $T_{i j}$ (unit $\mathrm{Nm}^{-2}$ ) have been represented in rows one to three, the angular momentum flux components $M_{i j}$ (unit $\mathrm{Nm}^{-1}$ ) in rows four to six. The numerical aperture of the focusing beam is kept in the paraxial range $\left(s_{0}=0.01\right)$.

The transition from low numerical aperture to high NA $\left(s_{0}=0.95\right)$ gives rise to a lack of circular symmetry in the separate electric or magnetic energy density. Electromagnetic cross-terms like the Poynting vector $\vec{S}$ and the linear and angular momentum densities, $\vec{m}$ and $\vec{j}$, maintain their circular 
symmetry; the flux components $T$ and $M$ do not have this property. When comparing the T-components in Figure 7 with its low-NA counterparts in Figure 6, it becomes apparent that especially the elements $T_{x x}, T_{x y}=T_{y x}$ and $T_{y y}$ have increased in relative value with respect to $T_{z z}$. The effect on the $M$-components at high NA is the appearance of substantial $z$-flux components in the $x$ - and $y$-direction. The net result is an in-plane tangential flow pattern of $z$-angular momentum, but the $z$-flow component of the $z$-angular momentum stays rigorously zero, neither is there net transport of $z$-angular momentum through a plane $z=$ constant.
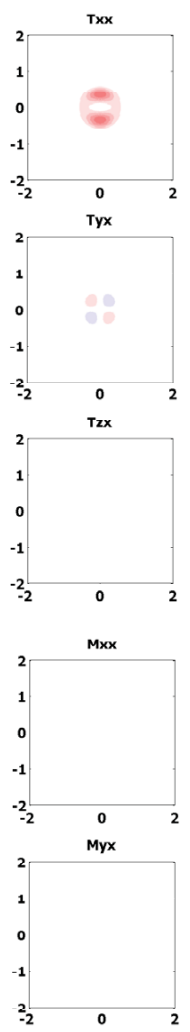

$\begin{array}{lc}-2 & 0 \\ -2 & 0 \\ M 2 x & \end{array}$

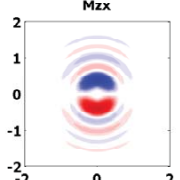

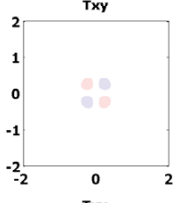

Try

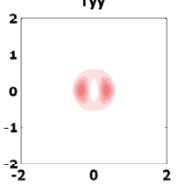

T<y
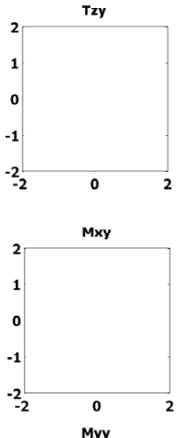

Myy

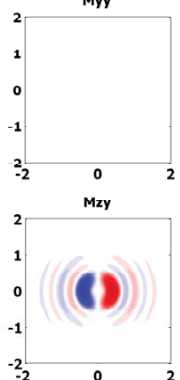

Mxis

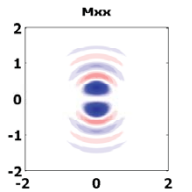

Myx

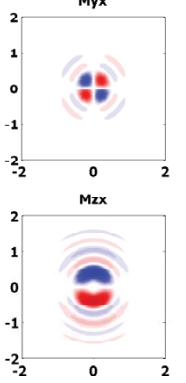

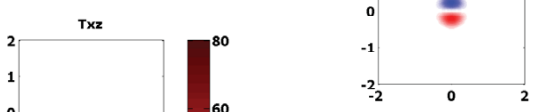
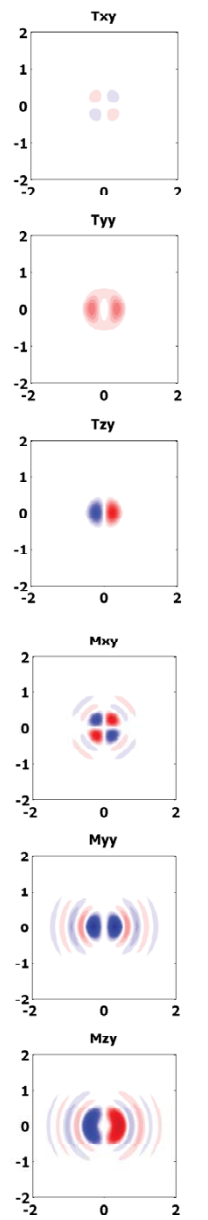
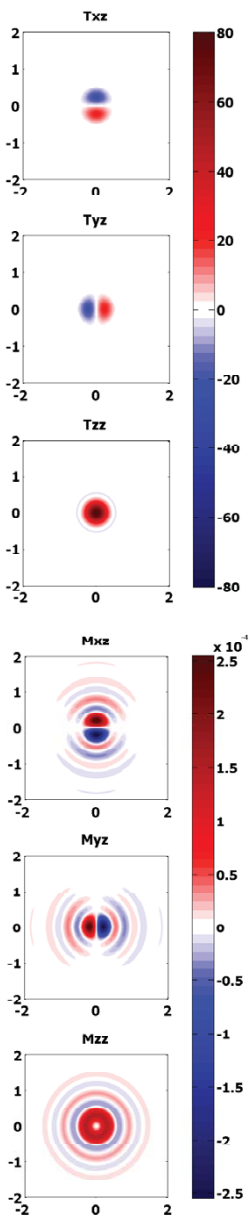

FIG. 8 Row one to three: graphs of the linear momentum flux components $T_{i j}$ in the nominal focal plane; row four to six: the $M_{i j}$-flux components in the same plane. The numerical aperture $s_{0}$ of the focusing beam amounts to 0.95 , left-handed circular polarisation, $(a, b)=(1, i) / \sqrt{2}$.

The Zernike coefficients defining the aberration phase $\Phi$ are $\alpha_{2}^{2}=\alpha_{2}^{-2}=\pi / 4$. This applies to astigmatism with the main axes of the astigmatic wavefront deformation along the $x$ - and $y$-axis. The corresponding $\beta$-coefficients defining the pupil function are calculated using again a third order Taylor series expansion of the pupil phase function $\exp (i \Phi)$. The defocused image plane settings have been chosen such that they correspond to the focal line positions as predicted by geometrical optics aberration theory. Figure 9 shows the moduli of the electric field components (rows one to three, for defocus values $f=+\pi, 0-\pi$, respectively) and their phases (rows four to six).

$(a, b)=(1, i) / \sqrt{2}$, see Figure 8 . Regarding the $T$ - flux components, the main difference with the linearly polarised incident beam is found in the nonzero $T_{z x^{-}}, T_{z y^{-}}, T_{x z^{-}}$and $T_{y z^{-}}$ components. This is equivalent to a tangential flow pattern of $z$ - linear momentum, on top of the main flow component $T_{z z}$. The main difference with linear incident polarisation for the $M$-components is the appearance of an $M_{z z}$-component, created by the nonzero linear momentum components $T_{x z}$ and $T_{y z}$. This is the result of the spin momentum in the incident beam.

The next example in Figure 9 is a linearly polarised incident beam, $(a, b)=(1,0)$, suffering from astigmatic aberration.
The $T$ flux components (Figure 10, first three rows) show a corresponding effect, for instance regarding the components $T_{z x}$ and $T_{z y}$. An interesting effect of an astigmatic focused beam is found when inspecting the $M_{z z}$-component. Although the total integrated $z$-angular momentum flux is zero over the focal plane, it is possible to spatially select diagonal portions of the beam that carry specific $z$-angular momentum. The splitting comes more important when the astigmatic aberration is increased. In this way, it is possible to create selective angular momentum that propagates in the z-direction; the disadvanin plus and minus $z$-angular momentum in the focal plane be- 

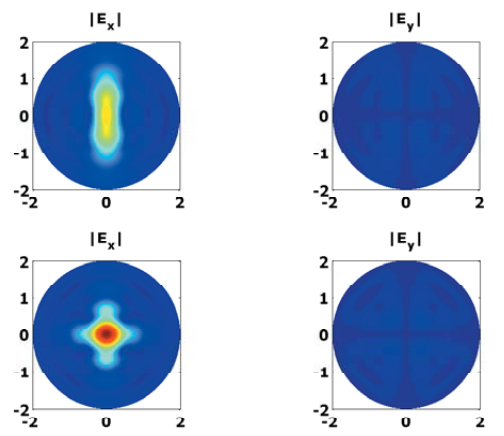

$\left|E_{y}\right|$

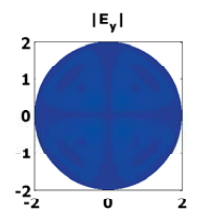

$\left|E_{y}\right|$
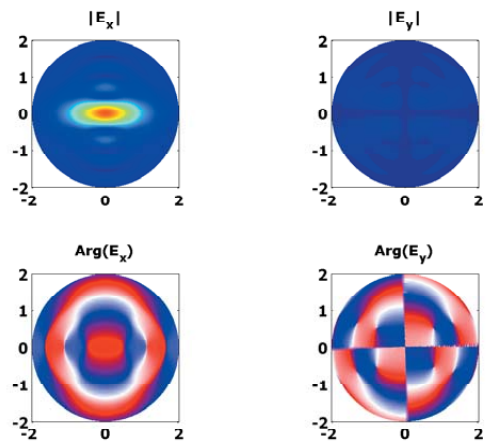

$\operatorname{Arg}\left(E_{\mathbf{x}}\right)$

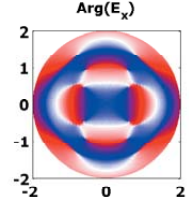

$\operatorname{Arg}\left(E_{\mathbf{x}}\right)$
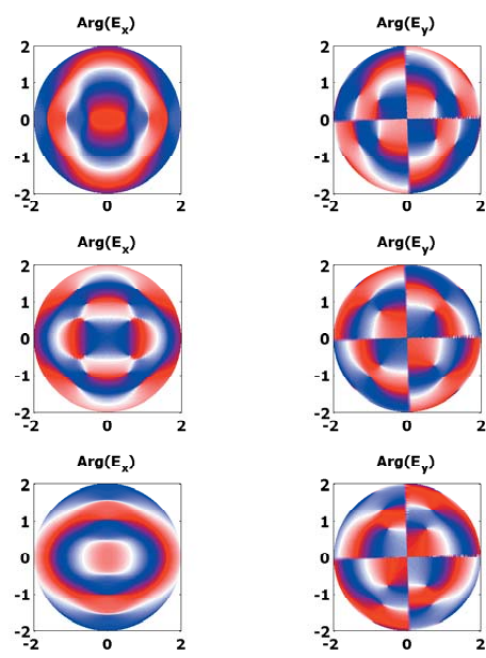

$\operatorname{Arg}\left(E_{\mathbf{y}}\right)$

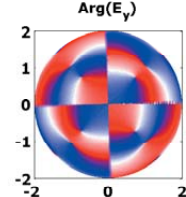

$\operatorname{Arg}\left(\mathrm{E}_{\mathrm{y}}\right)$

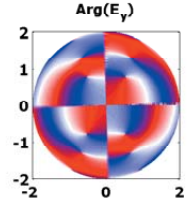

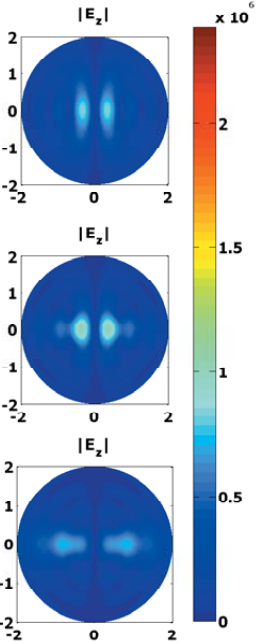

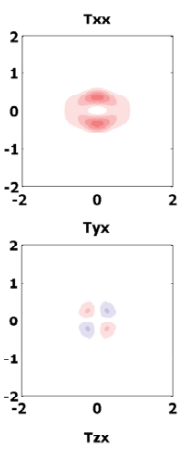

Tzx

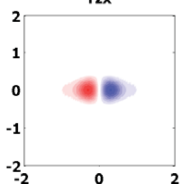

Mxx

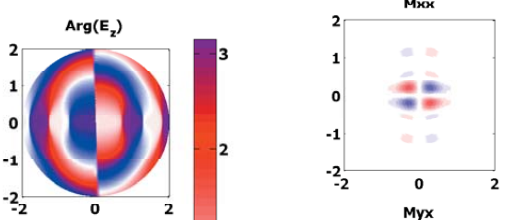

Myx

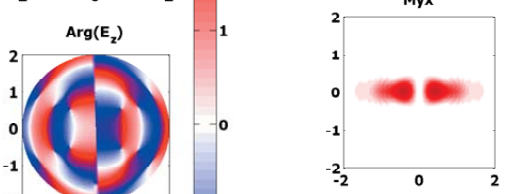

$-2$

0
$\operatorname{Arg}\left(E_{z}\right)$

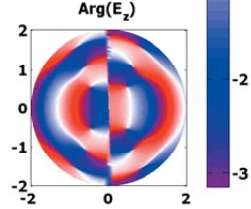

FIG. 9 The moduli (upper three rows) of the electric field components and their phases (lower three rows) in three image plane settings $(f=+\pi, 0,-\pi)$ for a linearly polarised beam, $(a, b)=(1,0)$, with astigmatic wavefront aberration characterised by the Zernike phase aberration coefficients $\alpha_{2}^{2}=\alpha_{2}^{-2}=i \pi / 4$, NA-value $s_{0}=0.95$.

tage is the loss-in-resolution that is accompanied by the increase in wavefront aberration.

The final example applies to a helical phase beam. Normally, these beams are described by Gauss-Laguerre modes as solutions of the paraxial wave equation. Here, we use the Zernike wavefront aberration representation in complex form and achieve a helical wavefront shape by putting $\beta_{1}^{1}=1, \beta_{0}^{0}=$ $\beta_{1}^{-1}=0$. The substitution in Eq.(4) yields a pupil function proportional to $\rho \exp (i \theta)$ that fits on a circular pupil with a sharp edge. In this sense, it is a more realistic pupil function realisation than an infinitely extending Gauss-Laguerre mode. Figure 11 clearly shows the doughnut-cross-section and the spiral phase of the $x$-component of the electric field. The component $E_{z}$ out-of-focus, normally composed of two lobes along the $x$ axis in phase opposition, is now transformed into an $S$-shaped profile with a maximum on the axis. This is due to the fact that the cancellation of the $z$-field component by geometrically opposite vector contributions is now turned into a constructive superposition because of the helical phase function. The absence of lateral components and the sole presence of a $z$ oriented electric field component in the beam centre explains the doughnut-like flow pattern of the axial component of the
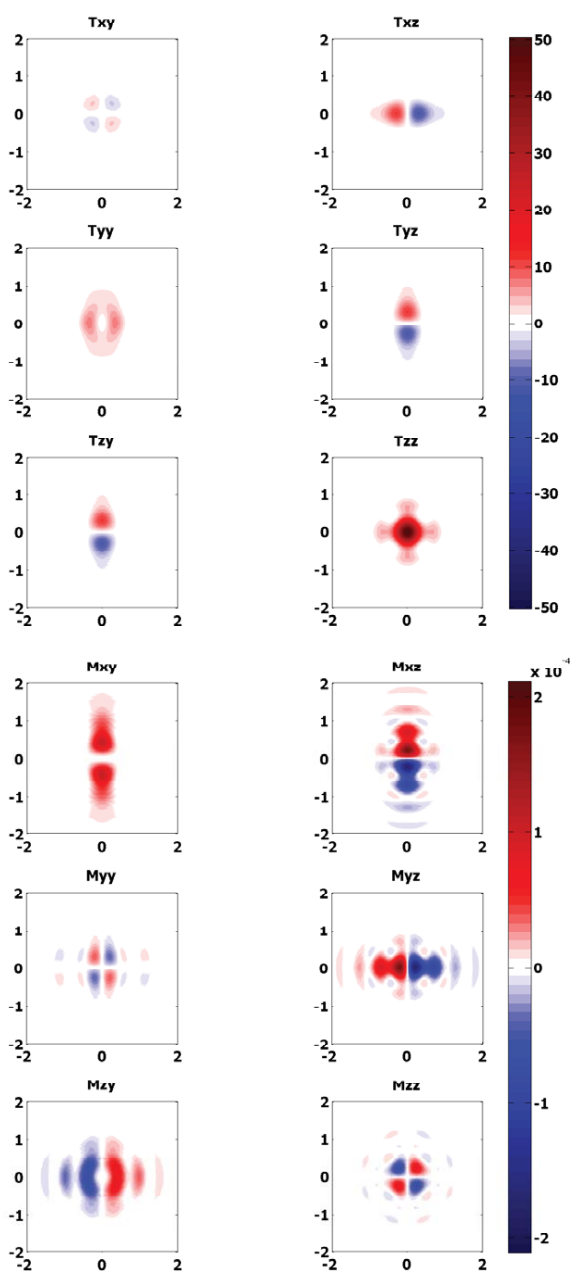

FIG. 10 Row one to three: graphs of the linear momentum flux components $T_{i j}$ in the nominal focal plane. Row four to six: the $M_{i j}$-flux components in the same plane. Same value of numerical aperture and astigmatic aberration as in Figure 9.

Poynting vector (see Figure 12, top row). In the same figure, row two to four, we show the $T$-flux components, that have a close resemblance with the $T$-components in Figure 8, both introducing a helical flow pattern of momentum in the beam. The main difference is found in the $T_{z z}$-component that has a doughnut-shape in the case of the helical phase aberration. Inspection of the $M$-flux components for both cases (left-handed circular polarisation, see Figure 8, and helical phase according to an $\exp (+i \theta)$-function, Figure 12, row five to seven), shows a very close resemblance; it can also be concluded that the sign of the angular momentum is identical in both cases.

\section{CONCLUSION}

The energy and momentum flow in the focal region of a high-numerical-aperture beam has been described using the extended Nijboer-Zernike diffraction theory. The analytic Gfunctions with indices $k l,|k|,|l| \leq 2$, characteristic for the through-focus intensity distribution in the presence of aberrations and defined in an earlier publication to represent the electric energy density, were used here to describe the Poynting vector, the momentum densities and the corresponding momentum flow components. 

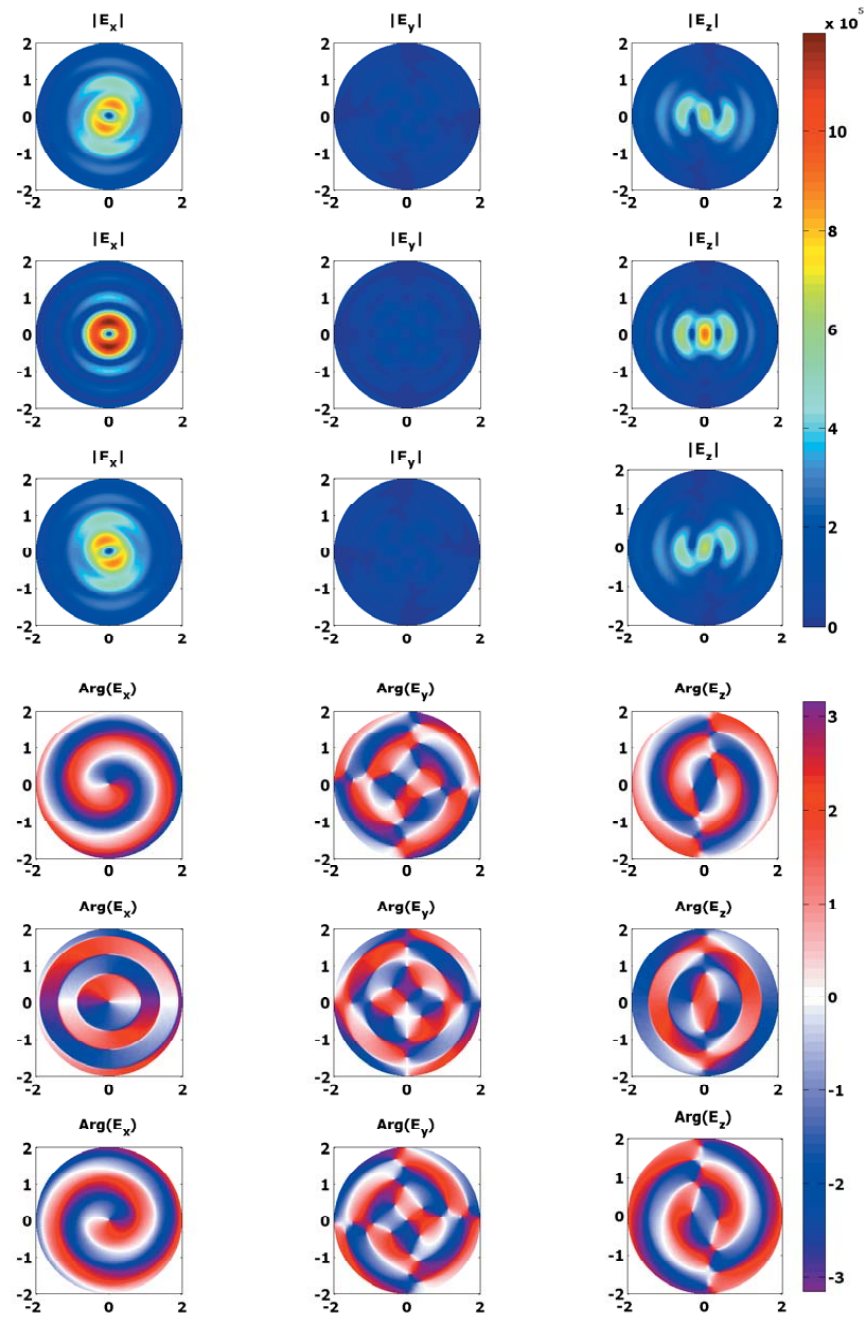

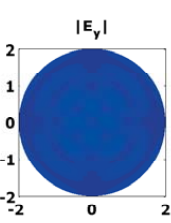

$\left|F_{y}\right|$
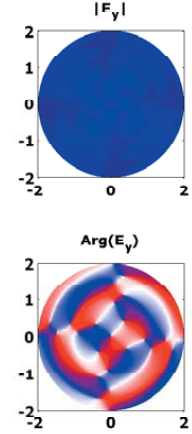

$\operatorname{Arg}\left(E_{v}\right)$

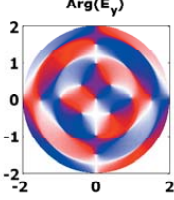

$\operatorname{Arg}\left(E_{\mathrm{y}}\right)$

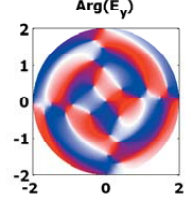

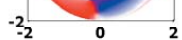

$\operatorname{Arg}\left(E_{z}\right)$

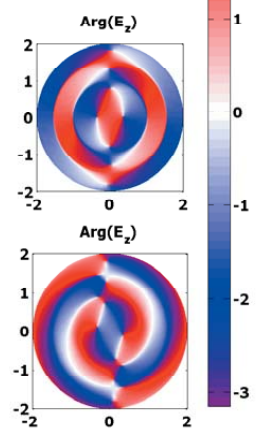

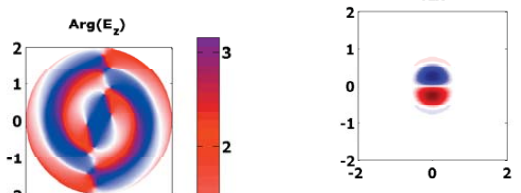

FIG. 11 The moduli (row one to three) of the electric field components (unit $\mathrm{Vm}^{-1}$ ) and their phases (row four to six) in three image plane settings $(f=-\pi, 0,+\pi)$ for a helical phase linearly polarised beam, $(a, b)=(1,0)$, with the single Zernike coefficient $\beta_{1}^{1}=1$, NA-value $s_{0}=0.95$.

Special attention has been paid to the angular momentum representation because its description at high numerical aperture is the subject of a discussion on what would be the optimum representation of angular momentum, going from the paraxial to the high-aperture case. Using the expression for the $M_{z z}$-component of the angular momentum that directly follows from the Maxwell stress tensor of linear momentum, we find an expression with a subdivision in angular momentum of both spin and orbital origin. The way in which the momentum of spin and orbital origin are added in the focal region depends on the position in the diffraction image according to Eq.(43). This is due to the fact that the point-spread functions belonging to a beam with only spin momentum or only orbital momentum are fundamentally different. A mixture of both sources of momentum in a focused beam gives rise to the nonlinear addition of momentum within the resulting point-spread function including cross-terms. We also have to conclude that the angular momentum component $M_{z z}$ does not show a fundamentally different behaviour for low- or high-numerical-aperture focused beams. The effect of a high numerical-aperture is to introduce a correction term with a relative value given by the aperture squared. Another way of splitting angular momentum in a spin contribution and an
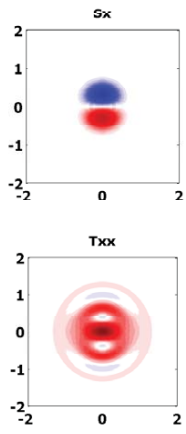

Tyx

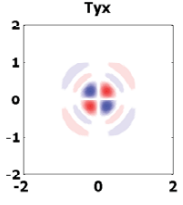

$\operatorname{Tzx}$
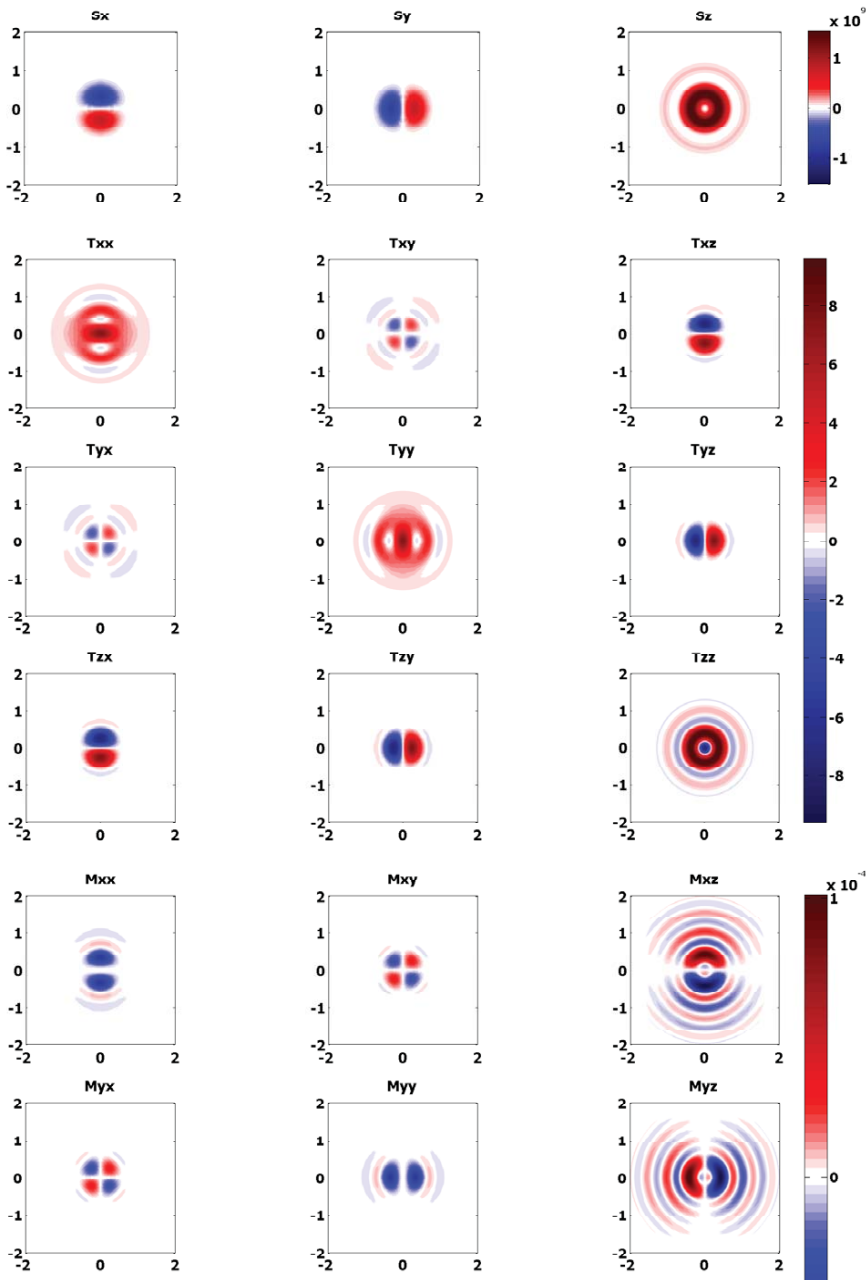

Myy
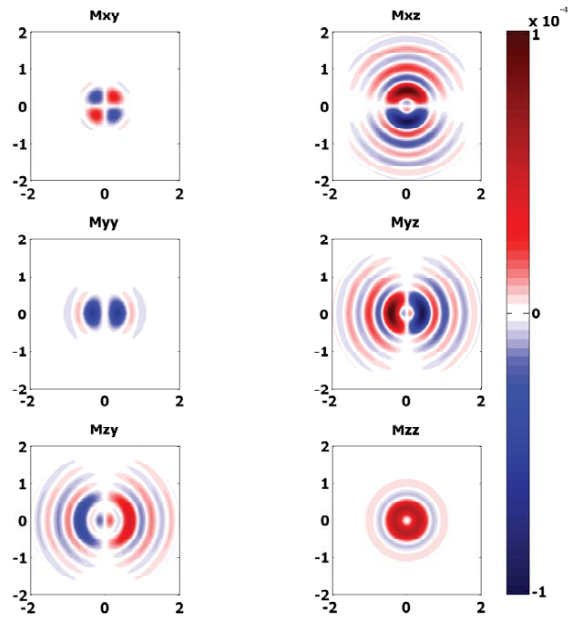

FIG. 12 First row: the components of the Poynting vector in the nominal focal plane. Row two to four: graphs of the linear momentum flux components $T_{i j}$ in the nominal focal plane and the $M_{i j}$-flux components in the same plane (row five to seven). Same numerical aperture, state of polarisation and value of helical phase term as in Figure 11.

orbital contribution has been proposed by Barnett, Eqs.(47) and (52), and his model leads to extra high numerical aperture terms that have a relative importance given by the aperture value to the fourth power. Cross terms with this relative weight arise between the spin and orbital contributions. Finally, we have presented some numerical examples that illustrate the intricate behaviour of the momentum flow components in a high-NA focused beam. It was particularly interesting to observe the close resemblance in angular momentum flow between a focused beam with spin momentum and a beam with orbital angular momentum.

\section{ACKNOWLEDGEMENT}

The authors would like to thank an anonymous reviewer of the journal for valuable comments and suggestions for improvement of the manuscript. 


\section{References}

[1] J. J. M. Braat, P. Dirksen, A. J. E. M. Janssen, and A. S. van de Nes, "Extended Nijboer-Zernike representation of the vector field in the focal region of an aberrated high-aperture optical system" J. 0pt. Soc. Am. A 20, 2281-2292 (2003).

[2] W. Ignatowsky, "Diffraction by a lens of arbitrary aperture," Trans. Opt. Inst 1, 1-36 (1919).

[3] B. Richards and E. Wolf, "Electromagnetic diffraction in optical systems II. Structure of the image field in an aplanatic system" P. Roy. Soc. A: Math. Phy. 253, 358-379 (1959).

[4] M. Mansuripur, "Distribution of light at and near the focus of high-numerical-aperture objectives" J. 0pt. Soc. Am. A 3, 20862093 (1986).

[5] M. Mansuripur, "Certain computational aspects of vector diffraction problems" J. Opt. Soc. Am. A 6, 786-805 (1989).

[6] R. Kant, "An analytical solution of vector diffraction for focusing optical systems" J. Mod. Optic. 40, 337-348 (1993).

[7] R. Kant, "An analytical solution of vector diffraction for focusing optical systems with Seidel aberrations" J. Mod. Optic. 40, 22932311 (1993).

[8] C. J. R. Sheppard and P. Török, "Efficient calculation of electromagnetic diffraction in optical systems using a multipole expansion" J. Mod. Opt. 44, 803-819 (1997).

[9] S. Stallinga, “Axial birefringence in high-numerical-aperture optical systems and the light distribution close to focus" J. 0pt. Soc. Am. A 18, 2846-2859 (2001).

[10] S. Stallinga, "Light distribution close to focus in biaxially birefringent media" J. Opt. Soc. Am. A 21, 1785-1798 (2004).
[11] S. Stallinga, "Strehl ratio for focusing into biaxially birefringent media" J. Opt. Soc. Am. A 21, 2406-2413 (2004).

[12] A. J. E. M. Janssen, "Extended Nijboer-Zernike approach for the computation of optical point-spread functions" J. Opt. Soc. Am. A19, 849-857 (2002).

[13] J. J. M. Braat, P. Dirksen, and A. J. E. M. Janssen, "Assessment of an extended Nijboer-Zernike approach for the computation of optical point-spread functions" J. Opt. Soc. Am. A 19, 858-870 (2002).

[14] J. J. M. Braat, P. Dirksen, A. J. E. M. Janssen, A. S. Van de Nes, and S. Van Haver, "Extended Nijboer-Zernike approach to aberration and birefringence retrieval in a high-numerical-aperture optical system" J. Opt. Soc. Am. A. 22, 2635-2650 (2005).

[15] J. J. M. Braat, S. van Haver, A. J. E. M. Janssen, and P. Dirksen, "Assessment of optical systems by means of point-spread functions" in Progress in Optics, ed. E. Wolf, 51, Chapter 6, (Elsevier Publishing, Amsterdam).

[16] J. D. Jackson, Classical Electrodynamics (John Wiley and Sons, New York, 1999).

[17] S. M. Barnett, "Optical angular momentum flux" J. Opt. B: Quantum S. 0. 4, S7-S16 (2002).

[18] C. van der Avoort, J. J. M. Braat, P. Dirksen, A. J. E. M. Janssen, "Aberration retrieval from the intensity point-spread function in the focal region using the extended Nijboer-Zernike approach" J. Mod. Optic. 52, 1695-1728 (2005).

[19] F. Nicolas, S. Coëtmellec, M. Brunel, D. Allano, D. Lebrun, and A. J. E. M. Janssen, "Application of the fractional Fourier transformation to digital holography recorded by an elliptical, astigmatic Gaussian beam" J. Opt. Soc. Am. A 22, 2569-2577 (2005). 